\title{
Effects of oxygen ozone therapy on cardiac function in a patient with a prior myocardial infarction
}

\author{
Sergio Pandolfi, ${ }^{1,2}$ Angelo Zammitti, ${ }^{1,2}$ Marianno Franzini, ${ }^{1,3}$ Vincenzo Simonetti, ${ }^{1,4}$ William Liboni, ${ }^{5}$ \\ Luigi Valdenassi, ${ }^{1,3}$ Filippo Molinari ${ }^{6}$ \\ ${ }^{1}$ Scientific Society of Oxygen-Ozone Therapy, Gorle (BG); ${ }^{2}$ Villa Mafalda Nursing Home, Rome; ${ }^{3}$ Department of \\ Internal Medicine and Medical Therapy, Master in Oxygen-Ozone Therapy, University of Pavia; ${ }^{4}$ Kaos non-profit \\ Association, Caselle Torinese (TO); ${ }^{5}$ Un Passo Insieme Onlus Foundation, Valdellatorre (TO); ${ }^{6}$ Department of \\ Electronics and Telecommunications, Politecnico di Torino, Turin, Italy
}

\begin{abstract}
Oxygen-ozone therapy has been piloted in Italy patients with ischemic heart disease or suffering from myocardial infarction since 1991. Over time, it has been observed that ozone exerts a significant anti-inflammatory and rheological activity: ozone activates the redox system, lowers the pro-inflammatory cytokines $16 \beta$, TNF $\alpha$, modulates the NF-KB system, reduces platelet aggregation, and stimulates the release of growth factors. It is because of these characteristics that oxygen-ozone therapy is used in the prevention and treatment of ischemic heart disease and for post-infarction rehabilitation. The case study - a critically ill patient aged 76 with acute myocardial infarction (AMI), suffering from ischemic heart disease and heart attacks, high blood pressure, renal failure, ischemic vascular pluri-infarct CNS disease, Parkinsonism, stroke, neurological bladder disease and debilitating inguinal hernia - after a year and a half of AMI with infusions of autologous blood treated with ozone (GAE), recovered the left ventricular ejection fraction from 33 to $50 \%$ and underwent a successful inguinal hernia operation. It appears evident that oxygen ozone therapy using GAE protect the hearts of patients suffering from ischemic heart disease, and is useful during the acute phase of infarctions, as well as for rehabilitating patients who have had an AMI with stenting.
\end{abstract}

Correspondence: Sergio Pandolfi, Scientific Society of Oxygen-Ozone Therapy, via Roma 69, Gorle (BG), Italy.

Tel/Fax: +39.035.300903.

E-mail: sergiopandolfis2@gmail.com

Key words: Oxygen ozone therapy; Cardiac function; Myocardial infarction; Autologous blood treated with ozone (GAE).

Received for publication: 12 April 2017

Accepted for publication: 19 April 2017

(C) Copyright S. Pandolfi et al., 2017

Licensee PAGEPress, Italy

Ozone Therapy 2017: 2:6745

doi:10.4081/ozone.2017.6745

This article is distributed under the terms of the Creative Commons Attribution Noncommercial License (by-nc 4.0) which permits any noncommercial use, distribution, and reproduction in any medium, provided the original author(s) and source are credited.

\section{Introduction}

Oxygen ozone therapy in patients with ischemic heart disease or had suffered acute myocardial infarction (AMI), was first tested in Italy at the end of 1991, when Prof. B. Lettieri, Professor of Anaesthesia and Reanimation at the Federico II of Naples treated several patients with ozonised autologous blood infusions during the acute phase of myocardial infarction ${ }^{1}$ and obtained favourable results in terms of pain and prognosis. In 1996, oxygen-ozone therapy was used in the prevention of recurrent AMI, and significant protection from re-infarction was demonstrated. ${ }^{2}$

Ozone exerts an important anti-inflammatory and rheological activity, despite being a strong oxidant, via its paradoxical effect of activating the cellular antioxidant system, ${ }^{3}$ which modulates the functioning of endogenous cellular enzymes that protect cells against free radicals, and increases DNA transcription at the level of antioxidant enzymes. It activates the Redox system, lowering the pro-inflammatory cytokines $1 \beta, 6 \mathrm{TNF} \alpha,{ }^{4}$ modulates the NF$\mathrm{KB}$ system, ${ }^{5}$ reduces platelet aggregation ${ }^{6}$ and stimulates the release of various growth factors. ${ }^{6,7}$

Due to these characteristics, oxygen-ozone therapy was used for the prevention and treatment of ischemic heart disease and in postinfarct rehabilitation. The ejection fraction of the left ventricle remained under 33\% from January 2014 to March 2015, despite coronary angioplasty and medical treatment. After a year and a half of AMI, the patient started the oxygen-ozone therapy using GAE, he recovered the left ventricular ejection fraction, from 33 to $50 \%$, and successfully underwent inguinal hernia surgery.

\section{Case Report}

The case report examines the outcome of treatment with oxygen-ozone therapy in a 76-year-old critically ill patient, with a previous myocardial infarction, suffering from ischemic heart disease and myocardial infarction (January 2014), high blood pressure, kidney disease, parkinsonism, ischemic cerebrovascular pluriinfarcts, brain ictus, a neurological bladder and a debilitating inguinal hernia. The ejection fraction of the left ventricle was assessed by repeated echocardiographic examinations, and remained under 33\% from January 2014 to March 2015, despite coronary angioplasty and medical treatment.

From 3 July 2015, the patient was subjected to an oxygen ozone therapy cycle via 2 autologous infusions of ozonized blood 
(GAE) on a weekly basis. During the first two months of therapy, the patient showed a marked improvement in cardiac status with a reduction in asthenia and improvement in neurological status. The improved cardiac status was confirmed by an echocardiogram on $05 / 11 / 2015$, which showed an increase in the left ventricular ejection fraction of $33-50 \%$. In this case, the improvements in cardiac function and left ventricular EF were detected only after starting systemic oxygen ozone therapy using GAE. In April 2015, before starting the oxygenated ozone therapy, the inguinal hernia had been assessed as inoperable in view of the low cardiac status, while at the next reassessment in December 2015, after having been subjected to oxygen ozone therapy for five months, it was judged operable due to improvements in cardiac function. On February 19, 2016 the inguinal hernia was successfully operated on. Administration of oxygen-ozone was performed via GAE, using bags approved for oxygen ozone therapy, in accordance with EC regulations.

The ozone generator used was a Multiossigen Medical 95, EEC 93/42 certified in class $2 a$. For each procedure $200-220 \mathrm{~mL}$ of blood were collected, and treated with $200 \mathrm{cc}$ of a mixture of ozone in oxygen at a concentration of 40 micrograms of ozone.

\section{Discussion}

Ozone is a gas characterised by its instability, which is naturally present in the body and the atmosphere. Its administration into an organism leads to an initial, transient oxidative stress that the redox systems of the blood and plasma work together to counteract. Obviously the concentrations of ozone used therapeutically never exceed the antioxidant potential of the blood. ${ }^{8-10}$

When placed in contact with the plasma, ozone reacts instantly with the antioxidant systems (especially uric acid, ascorbic acid, glutathione, cysteine and albumin), therefore, only a small part of the initial dose reacts with polyunsaturated fatty acids (PUFAs).

Therefore, the potential energy of the ozone is transferred to two main messengers, such as $\mathrm{H}_{2} \mathrm{OR}_{2}$ and the aldehyde molecules 4hydroxynonenal (4HNE) and trans-4-hydroxyhexenal (HHE). Given the high reactivity of ozone, these reactions take place within a few seconds, and a few minutes of contact between blood and the mixture of oxygen-ozone is generally sufficient, because ozone is completely exhausted and the oxygen saturates the haemoglobin system, dissolving into the plasma. The antioxidant systems are mainly replenished within 20 minutes of exposure to the gas mixture.

De-ionised $\mathrm{H}_{2} \mathrm{OR}_{2}$ quickly enters all of the blood cells and induces the following changes: in active erythrocytes, glycolysis, increasing intracellular concentrations of ATP and 2, 3 DPG. This translates into a right shift of the haemoglobin dissociation curve, which therefore more readily releases oxygen into the peripheral tissues. ${ }^{10}$ In leukocytes, the phagocytic activity of neutrophils is increased. In lymphocytes and macrophages, intracellular LKB patterns, one of the components of NFkB are activated. The trimer, which is degraded in proteasomes into the hetero-dimers p50-p65, can activate the transcription of over 100 genes. Interleukins are produced, as are various acute phase proteins, as well as IFN and TNF $\alpha .{ }^{11}$ In platelets, PDGF AB, TGFB1 and growth factors are produced.

The aldehydes react with GSH, carnosine and albumin in particular, and are transported into various body tissues. The toxicity of $4 \mathrm{HNE}$ and HHE is contrasted by compensatory mechanisms like detoxification, dilution and excretion; these compounds, in fact, recognized as oxidizing agents, induce a response from the body that produces SOD, haem-oxygenase, G6PDH and other powerful antioxidants. The aldehydes also stimulate the production of endothelial Nos, ${ }^{12}$ improving peripheral perfusion and tissue oxygenation. In addition, in a randomised clinical study of 140 patients, of which 70 were subjected to oxygen ozone therapy and 70 were a control group, blood chemistry parameters showed a decrease in Reactive Oxygen Metabolites (300 \pm 10.1 UCARR at 12 months compared to an initial value of $380 \pm 10.4$ UCARR, $\mathrm{P}<0.05$ ) and an increase in plasma Biological Antioxidant potential values (2100 \pm 34.8 micromoles/vitamin C after 12 months compared to an initial value of $1610 \pm 36.2, \mathrm{P}<0.05$ ) in treated patients compared to the control group. ${ }^{13}$

These data demonstrate that ozonized blood haemoinfusions play a role in reducing oxidative stress by endogenously stimulating the production of antioxidant enzymes.

After input from Prof. Lettieri in $1991^{1}$ and $1996^{2}$ several studies on mice were conducted: when subjected to myocardial ischemia, rats pre-treated with oxygen ozone therapy showed smaller ischemic areas and improved cardiac function indexes than those that were not treated with ozone. ${ }^{12-14}$ As for in vivo studies, in 2008 the ACCLAIM trial was published; ${ }^{15}$ this was a randomised doubleblind study in which NYHA II-IV patients were given ozonized blood using the Celacade System, which provides small amounts of blood (10-20 ml) that have been exposed to large concentrations of ozone, which is then processed and heated at $42^{\circ} \mathrm{C}$ under UV light, and then re-injected into the buttock, and is comparable to a small auto haemoinfusions of ozonised blood. The study showed a correlation between administering this treatment, oxygen ozone therapy, which is described as non-specific immuno-modulatory, and clinically assessed events like death from any cause, hospitalisation for cardiac reasons, the need for i.v. therapy for heart failure etc. The results, however, favoured treatment with ozone, despite an incomplete therapeutic procedure being performed and not having performed GAE (large auto haemotherapy) that are indicated in all diseases of the organs, microcirculation and ischemias. The scientific community ${ }^{16}$ has questioned using a method like Celacade for ozone therapy, since the high concentration, when used on a small blood samples, exceeds the redox potential of the plasma, whilst the heat and UV rays it uses denature most of corpuscular components. ${ }^{16,17}$

In another study, the effects of acute administration of a mixture of oxygen/ozone $\left(\mathrm{O}_{3}\right)$ were assessed in damaged myocardial tissue following an ischemic event. ${ }^{18}$ The study was performed on rats subjected to acute myocardial ischaemia and reperfusion. The animals were treated with an oxygen-ozone mixture of 100,150 and $300 \mathrm{micrograms} / \mathrm{kg}$ injected intraperitoneally one hour before the ischemic damage.

Infarct size, markers of necrosis and tissue damage, nitro tyrosine, CD68, CD8, CD4 and caspase-3 levels were measured. The results showed a smaller sized area infarction in rats pre-treated by insufflation with the oxygen-ozone mixture, and a parallel reduction in tissue levels of nitro tyrosine, inflammatory markers (CD68) and of the immune response (CD8 and CD4). These data indicated that the damage associated with ischemia and myocardial reperfusion could be counteracted by pre-treatment with systematically administered gaseous mixtures of oxygen-ozone.

Other more recent studies have shown that after a myocardial infarction, the level of endothelial progenitor cells in the myocardium is reduced. These cells are derived from bone marrow, and serve to mobilise, migrate and differentiate into endothelial cells in situ, forming a mobile cellular reserve that can repair endothelial damage. Strategies to increase the CEP in an ischemic heart seem to improve the neo vascularisation of ischemic tissue, and may improve myocardial blood flow by decreasing ischemic damage. One experimental study in animals ${ }^{18}$ showed that oxygen/ozone treatment protected the heart from AMIs thanks to a localised 
increase in eNOS activity, and the recruitment of endothelial progenitor cells.

The ability of oxygen ozone therapy to lower re-stenosis rates after implanting metal stents in pigs due to its antioxidant and antiinflammatory properties has recently been assessed. ${ }^{19}$ The recurrence of the stenosis, re-stenosis, or the lack of re-endothelialisation can occur after percutaneous trans-luminal coronary angioplasty, even when medicated drug-eluting stents are used, due to inflammation and oxidative stress at an endothelial level. Twelve male Landrace pigs $(51 \pm 9 \mathrm{~kg})$ were subjected to percutaneous transluminal metal stenting in the circumflex coronary arteries under infusion of heparin with fluoroscopic guidance using standard techniques. This randomised study was conducted with 6 pigs subjected to ozone-oxygen therapy and the other 6 pigs receiving a placebo treatment. Before applying the stent ( 24 hours before) and twice a week for 30 days after stenting, venous blood was collected, ozonized and reinfused. The same procedure was performed in the placebo group, with the ozone treatment omitted. Both groups received an anticoagulant treatment. Histopathology and immunohistochemical assessments were performed. The results of the study showed a severe inflammatory reaction and restenosis, with an increase in the immunohistochemical expression of thioredoxin-1 in the placebo group, 30 days after the surgery. Contrarily, ozone therapy significantly reduced the inflammatory response and re-stenosis, and no immunohistochemical increase in Trx-1 expression was evident 30 days after surgery. The same immune marker for PRX-2 was negative in both groups. Surprisingly, ozonised autologous haemotherapy reduced re-stenosis 30 days after PTCA with BMS implantation in pigs. Stimulation of the redox system, superoxide dismutase, catalase, carnosine, albumins, haem-oxygenase and G6PDH due to the pretreatment with ozone neutralised the oxidative damage by increasing the post-ischemic antioxidant capacity, which reduced the damage, the need for antioxidant enzymes and the risk of re-stenosis with the stent.

It is interesting to note that data reported by Profs. Lettieri and Chiefari at the University of Naples ${ }^{1,2}$ also showed that essentially, the possibility of recovering myocardial tissue affected by infarction depended on the treatment used, in both the early hours of pain and in the phase of coronary reperfusion, because, at these times, the highest mortality rates are observed. The use of thrombolytics can reduce the necrotic area, but there is some evidence it can easy induce the onset of severe rhythmic disorders, immediately after the restoration of myocardial re-vascularisation in the territory affected by ischemia, due to toxic substances like oxygen free radicals, leukotrienes and bradykinins being formed and entering the circulation. The group of patients treated with oxygen-ozone therapy via large ozonised autologous haemotherapy showed a faster disappearance of pain; furthermore, the extent of the infarcted area in them was more limited, because it lowered the viscosity of the blood and improved its rheology (the flow of blood through the vessels). This allowed a significant increase in perfusion of the area surrounding the infarct at an early stage of the condition, thus achieving, via the capillaries, low blood viscosity and the recovery of perfusion in the at risk zone.

In this study, ${ }^{1}$ conducted by Prof. Lettieri et al., acute phase AMI patients were treated with oxygen-ozone therapy mediated through large auto haemoinfusions of ozonised blood. Two groups of infarcted patients were selected: 1) the first group was subjected to conventional treatment (group A); 2) the second group (group $\mathrm{B}$ ), in addition to conventional treatment, was subjected to oxygenozone-therapy, according to the following scheme: $150 \mathrm{cc}$ of blood were taken, mixed with oxygen-ozone and re-infused. The first treatment was administered within 20 minutes of admission to hos- pital, the second 48 hours afterwards and the third at 96 hours. Assessments regarding the extent of reperfusion were based on the occurrence, in relation to times, of the following events: 1) the disappearance or significant reduction of chest pain; 2) the reduction of the increased ECG ST-segment and; 3) the rapid release of serum CK-MB, with an early peak. Cardiac perfusion assessments are made on the basis of echocardiogram results, the possibility of myocardial tissue recovery, onset of an acute ischemic event, and are greatly influenced by behaviour therapy, used both in the first painful hours and at the coronary reperfusion stage. These are the most sensitive times in intensive treatment of these patients. This statement is supported by the international literature, which shows the highest death rates occur during in these two stages. The conclusions of large trials performed the 80 s opened a window of opportunity for reducing the necrotic area caused by an acute thrombotic process in one or more coronary branches, through the early use of thrombolytic agents, but, unfortunately, also highlighted how easy it is for severe rhythmic disorders to set in, immediately after restoring vascularisation to the myocardial territory affected by ischemia, due to the formation of toxic substances like oxygen free radicals, leukotrienes and bradykinins that enter the circulation. It has also been noted that the tissues surrounding the necrotic area, in the earliest hours following an infarct, are involved in a process of intense perifocal oedema, with onsite accumulation of $\mathrm{K}^{+}$and catecholamines: this phenomenon causes a typical action potential, described by Cranfield (Mount Kisko Hospital, NY, USA) as a slow response; in the area surrounding the focus, which is prone to oedema, and, more peripherally, to reactive ischemia, the impulse is, so to speak, sequestered in a portion of the conduction system, which allows normal perfusion to the surrounding tissues to unleash ectopic rhythms, peri-lesional electrical instabilities.

Weighing up the advantages and limitations of thrombolytic treatment in an attempt to achieve the dual objectives of reducing infarct size as it develops, and, at the same time, avoiding the establishment of dangerous arrhythmias as far as possible, Prof. Lettieri decided to render an original infarct treatment for infarction in the acute phase, by mixing ozone with a quantity of patient's blood and subsequently reinfusing it into the circulation at a very early stage, relative to the time the pain started. For this purpose, two homogeneous groups of patients were selected, both suffering from myocardial infarction, both observed within 8 hours of the painful symptomatology arising, and aged between 42 and 64 years. The first group was subjected to traditional therapy; the second to oxygen-ozone therapy, administered by auto haemo infusions of ozonised blood, before informed consent and clinical and instrumental diagnosis of the myocardial infarction.

In addition to conventional treatment the second group, designated $\mathrm{B}$, was subjected to ozonisation therapy, using following scheme: $150 \mathrm{cc}$ of blood were taken and mixed with ozone-oxygen, then the blood was re-infused into a vein, at a slow drip rate. The first treatment was carried out within 20 minutes of admission; the second 48 hours afterwards; the third at 96 hours. The infusion therapy was initiated depending on the individual needs of the patients, regardless of the double-blinding. Judgments about the extent of reperfusion were based on the occurrence, in terms of time relationships, of the following events: 1) the disappearance, or a significant reduction in chest pain; 2) the extent of to which the S-T segment elevation dropped, with a progressive trend towards the isoelectric line, or with values equal to or less than $50 \%$ of the baseline value; 3 ) how fast serum CKMB was released with an early peak (greater than or equal to $16 \mathrm{~h}$ ); if the infarction index enzymes stabilised within 16 hours it indicated there has been a good re-perfusion. 
A cardiac performance score was awarded, on the basis of the results of a two-dimensional echocardiogram. The global kinetic and ejection fraction were evaluated using the Simpson method. The kinetic segmental, which is an entirely subjective observation, was quantified using a score of $3=$ normokinesia to $-1=$ dyskinesia.

On comparing the two groups of patients with regard to pain, which is a sign of myocardial ischemia, the results showed that average values (at 24 hours from pain onset) for group A were 5.5 points, corresponding to moderate to severe pain; whilst in group $\mathrm{B}$, the score was 3 , corresponding to mild pain. At 36-hours postadmission, the scores for both groups showed no significant variations, settling down to a score of 2 (just appreciable value). Significant results did emerge in CK-MB kinetics. In group A, the peak on the enzymatic curve was observed to extend beyond 19 hours of pain in $20 \%$ of cases, whilst in group B, the percentage was 10 (a value assessed as persistent occlusion.)

An earlier fall in CK blood levels was observed, before 16 hours (having been re-perfused), in $60 \%$ of patients in group B, treated with oxygen-ozone therapy, compared to $40 \%$ of group A.

From these data, we can deduce that the extent of the infarction was more limited in the group treated with oxygen-ozone, compared to the group treated with traditional therapy. Comparing now the sum of the electrocardiographic derivations present in the ST segment before treatment using the methods already described, with the sum of derivations, which showed a $\mathrm{Q}$ wave of necrosis, three weeks after infarction, a relationship was observed in favour of group B. Yet the fibrinogen rate, from the time of hospitalisation until the seventh day, after three cycles of treatment with oxygenozone, was observed to decrease by $43.7 \%$ in group B compared to baseline, whilst in group A, a decrease of $18 \%$ was observed. Bearing in mind that mechanical events occur earlier than electrical events, echocardiographic monitoring was carried out using the calculated ejection fraction obtained from the relationship between cardiac output and end-diastolic volume; in the first day, almost overlapping values were observed in the two groups, until, on the twentieth day, a five-point difference between the two groups was attained, in addition to which, in the last echocardiogram for group $\mathrm{B}$, the ejection fraction value in toto approached normal. The encouraging findings emerging from this study confirmed the validity of autologous ozonised blood infusions in ischemic heart disease.

The clinical and instrumental results were evaluated, the beneficial rheological effect of oxygen-ozone-therapy, which result in a significant reduction of blood viscosity, both as regard to the deformation of corpuscular component of the red blood cell series, as well as of those referred to as coagulation factors. Various authors have, over the years, reported ozone causes structural changes to fatty acids, which are basic constituents of biological membranes, that increase the plasticity of red blood cells; and yet, this gas creates a barrier of negative electric charges. This double action would respectively allow greater diffusibility of blood and an improvement in the microcirculation.

At an early stage of the disease this phenomenon would allow a significant increase in perfusion of the area surrounding the necrotised zone: the zone which is, in the acute phase of myocardial infarction, facing a dual destiny: firstly the danger that reactive vasoconstriction and the presence of toxic substances released from the necrotising area will accentuate the ischaemia until it widens the infarct, with an aggravation of the prognosis quoad vitam; or, secondly, where, via preferential microcirculation channels, it is possible to attain blood of a lower viscosity and induce recovery of blood perfusion in the risk zone; both of which are phenomena that appear to occur during treatment with oxygen-ozone therapy. Finally, it may be advisable to check, by follow-up at a distance with a greater number of patients treated with ozone, the significance of the drop in blood fibrinogen and the prolongation of coagulation parameter times.

As regards the improvements in rheology attributed to the fibrinogen decrease in patients undergoing GAE, a preliminary study into the efficacy of oxygen-ozone therapy for preventing recurrent myocardial infarction by exploiting the rheological effect of ozone, showed that patients with a previous myocardial who were subjected to GAE had a reduced risk of AMI recurrence.

\section{Conclusions}

The clinical history of this patient, who, at 74 years of age (January 2014), suffered a myocardial infarction with parkinsonism, hypertension, chronic renal disease and dyslipidaemia as comorbidities, and received oxygen-ozone therapy using large autologous ozonized blood infusions (GAE) for 18 months, demonstrates that this method acts on the heart's functional recovery by improving myocardial contractility and protects against the risk of subsequent recurrences of AMI. The 76-year-old patient who was the subject of this work is currently in maintenance therapy receiving one GAE per week, using 200-220 g of blood exposed to a mixture of oxygen and ozone at concentrations of 40-50 $\mu \mathrm{g}$ of $\mathrm{O}_{3}$, and has shown an improvement in general condition, mood recovery, stabilisation of cardiac function and normalisation of renal parameters, with further improvement in ambulation after a voluminous inguinal hernia was removed, and stable Parkinson's. Clearly, oxygen ozone therapy using GAE protects the heart of patients suffering from ischemic heart disease, and is useful in the acute phase of infarctions, and for rehabilitating patients who have had an AMI with stenting.

\section{References}

1. Lettieri B, Chiefari M, Vicario C. Cardiopatie ischemiche ed ozono-terapia. Giornate Meridionali di Ossigeno Ozono Terapia, Sorrento 9-10 Marzo 1991. Available from: www. angelipaolo.it/pdf/pag27.pdf

2. Lettieri B. Efficacia dell'ozono terapia nella prevenzione della recidiva dell'infarto al miocardio. Acta Toxicol 1996;17:2-3.

3. Valdenassi L, Franzini M, Simonetti V, Ricevuti G. Ossigenoozono terapia: stimolo paradosso dell'ozono. Ozone Therapy 2016;1:2-4.

4. Chang JD, Lu HS, Chang YF, Wang D. Ameliorative effect of ozone on cytokine production in mice injected with human rheumatoid arthritis synovial fibroblast cells. Rheumatol Int 2005;26:142-51.

5. Huth KC, Saugel B, Jakob FM, et al. Effect of aqueous ozone on the NF-kappaB system. J Dent Res 2007;86:451-6.

6. Maslennikov OV, Sharov IG, Potekhina IP, et al. Effect of ozone therapy on hemostatic changes in patients with vascular atherosclerosis. Klin Med (Mosk) 1997;75:35-7.

7. Bocci V, Bocci V. Studies on the biological effects of ozone: 10. Release of factors from ozonated human platelets. Mediators Inflamm 1999;8:205-9.

8. Bocci V. Is it true that ozone is always toxic? The end of a dogma. Toxicol Appl Pharmacol 2006;216:493-504.

9. Bocci V, Zanardi I, Travagli V. Oxygen/ozone as medical gas mixture. A critical evaluation of the various methods clarifies 
positive and negative aspects. Medical Gas Research 2011;1:6.

10. Bocci V, Borrelli E, Travagli V, Zanardi I. The ozone paradox: ozone is a strong oxidant as well as medical drug. Med Res Rev 2009;29:646-82.

11. Viebahn-Hänsler R. The use of ozone in medicine: mechanisms of action. Munich; Odrei Publishers: 2003.

12. Di Filippo, Marfella R, Capodanno P, et al. Acute oxygenozone administration to rats protects the heart from ischemia reperfusion infarct. Inflamm Res 2008;57:445-9.

13. Borrelli E, Bocci V. Visual improvement following ozonetherapy in dry age related macular degeneration; a review. Med Hypothesis Discov Innov Ophthalmol 2013;2:47-51.

14. Merin O. Ozone administration reduces reperfusion injury in an isolated rat heart model. J Card Surg 2007;22:339-42.

15. Torre-Amione G. Results of a non-specific immunomodula- tion therapy in chronic heart failure (ACCLAIM trial): a placebo-controlled randomised trial. Lancet 2008;371:228-36.

16. Melchart D. Immunomodulation through autohemotherapy in chronic heart failure. Forsch Komplementmed 2008;15:230.

17. Fildes JE, Shaw SM, Yonan N, Williams SG. Nonspecific immunomodulation in chronic heart failure. Lancet 2008;371:2083.

18. Di Filippo C, Luongo M, Marfella R, et al. Oxygen/ ozone protects the heart from acute myocardial infarction through local increase of eNOS activity and endothelial progenitor cells recruitment. Naunyn Schmiedebergs Arch Pharmacol 2010;382:287-91.

19. Barone A, Otero-Losada M, Grangeat AM, et al. Ozonetherapy protects from in-stent coronary neointimal proliferation. Role of redoxins. Int J Cardiol 2016;223:258-61. 